\title{
Anti-inflammatory effects of polymethoxyflavones from citrus peels: a review
}

\author{
Xiaoqi Wanga, Shiming Li ${ }^{\mathrm{b}}$, Chia-Cheng Wei ${ }^{\mathrm{a}}$, Junqing Huang ${ }^{\mathrm{a}}$, Min-Hsiung Pan ${ }^{\mathrm{c}}$, \\ Fereidoon Shahidid and Chi-Tang $\mathrm{Ho}^{\mathrm{a}^{*}}$
}

\begin{abstract}
aDepartment of Food Science, Rutgers University, New Brunswick, NJ 08901, USA
${ }^{\mathrm{b} C o l l e g e}$ of Life Sciences, Huanggang Normal University, Hubei, 438000, China

'Institute of Food Science and Technology, National Taiwan University, Taipei 10617, Taiwan

dDepartment of Biochemistry, Memorial University of Newfoundland, St. John's, NL, A1B 3X9, Canada

${ }^{*}$ Corresponding author: Shiming Li, College of Life Sciences, Huanggang Normal University, Hubei, 438000, China; Chi-Tang Ho, Department of Food Science, Rutgers University, New Brunswick, NJ 08901, USA. E-mail: shiming@rutgers.edu; ctho@sebs.rutgers.edu DOI: $10.31665 / J F B .2018 .3150$
\end{abstract}

Received: August 25, 2018; Revised received \& accepted: September 16, 2018

Citation: Wang, X., Li, S., Wei, C.-C., Huang, J., Pan, M.-H., Shahidi, F., and Ho, C.-T. (2018). Anti-inflammatory effects of polymethoxyflavones from citrus peels: a review. J. Food Bioact. 3: 76-86.

\begin{abstract}
Inflammation is a non-specific kind of biological immune response of body tissues to any type of external or internal injuries, such as pathogens, irritants and immune stress reactions. There are two types of inflammation, namely acute and chronic. Acute inflammation starts and develops rapidly, and is aroused by various factors, including injuries, infection, toxins or immune reactions. Chronic inflammation usually lasts for an extended long period of time and results from elimination failure of acute inflammation, autoimmune disorders, various pathogens and pathogenic environments. Except for the damage itself, there exists a direct and intimate connection between chronic inflammation and various clinic common diseases, such as neurodegeneration, as well as metabolic and cardiovascular ailments. Citrus peel is a by-product generated in citrus juice processing. Polymethoxyflavones (PMFs) exist abundantly and almost exclusively in citrus peels, and their biological activities have been broadly investigated in recent years. PMFs have proven to possess potential inhibitory bioactivities towards a number of functional and immune diseases including inflammation. The two most abundant PMFs exhibiting prominent bioactivities in citrus peels are nobiletin and tangeretin, ubiquitously detected in various citrus species. In this review, the beneficial health effects and the underlying molecular mechanisms of ten main citrus PMFs were illustrated against numerous inflammatory diseases, including inflammatory bowel disease (IBD), neuroinflammation and organ inflammation, among others.
\end{abstract}

Keywords: Citrus peel extract; Polymethoxyflavone; Anti-inflammation; Nuclear factor-kappa B (NF-кB); Disease conditions.

1. Introduction

Polymethoxyflavones (PMFs) exist exclusively and abundantly in citrus peels and have broadly been investigated in recent years resulting from their potential biological activities against various functional and immune disorders in human body, such as inflammation, cancer, neurodegeneration and metabolic syndrome, among others. In this contribution, the detailed health-promoting effects and the underlying molecular mechanisms of ten main citrus PMFs against numerous inflammatory diseases are reviewed.

\subsection{Molecular mechanism for inflammation}

The molecular mechanism of inflammation is illustrated in Figure 1. 


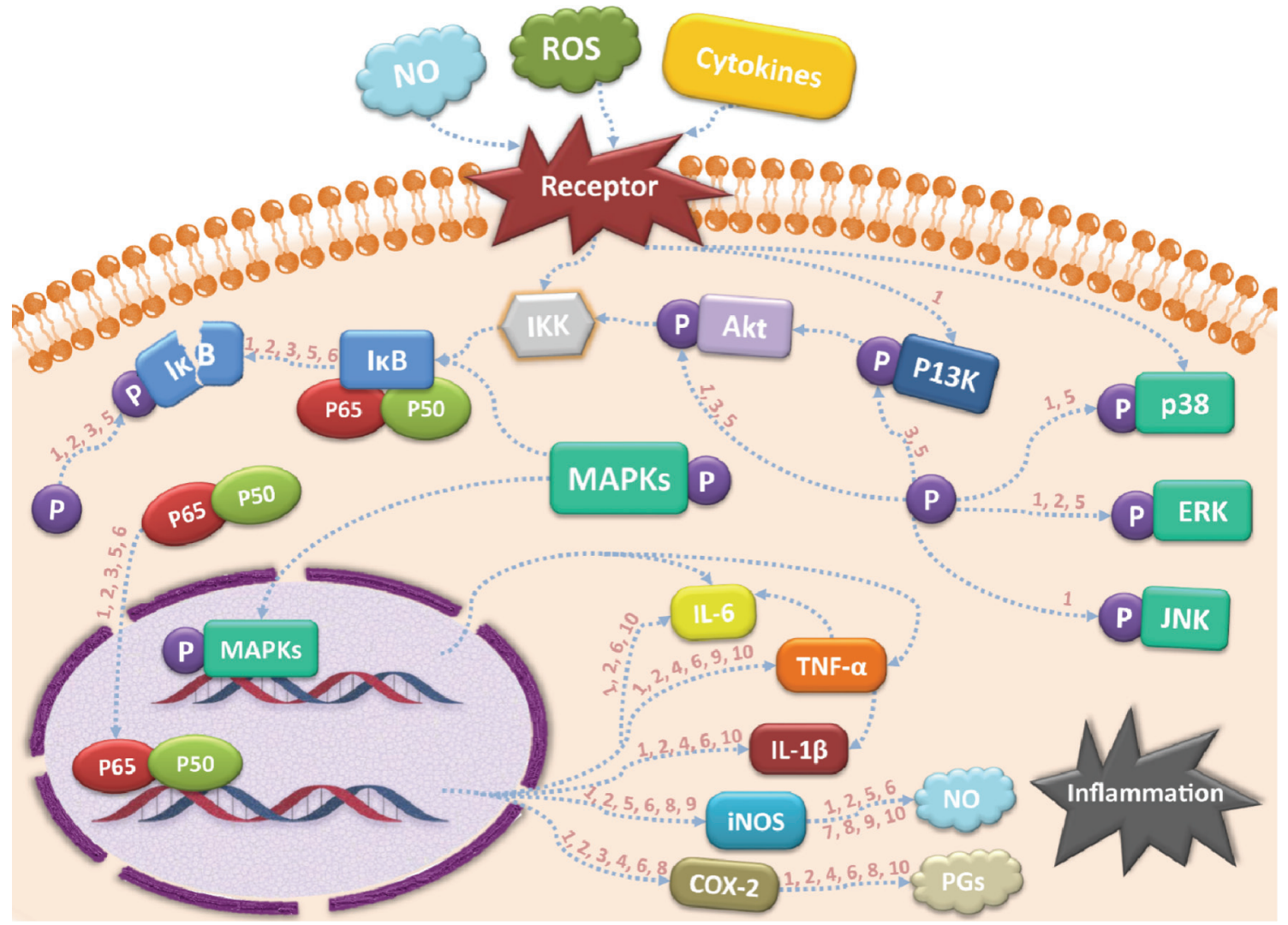

Figure 1. Possible anti-inflammatory signaling pathways of citrus PMFs. Numbers refer to different compounds: (1) Nobiletin; (2) Tangeretin; (3) 5-Demethyltangeretin; (4) 3,5,6,7,8,3', $4^{\prime}$-Heptamethoxyflavone; (5) 5-Hydroxy-3,6,7,8,3', $4^{\prime}$-hexamethoxyflavone: (6) Sinensetin; (7) Pentamethylquercetin; (8) 5-Hydroxy-3,7,3', $4^{\prime}$-tetramethoxyflavone; (9) Sudachitin; (10) Tetramethyl-O-scutellarin.

Being the crucial junction in inflammatory response, NF- $\mathrm{B}$ (nuclear factor kappa B) plays a key role as backstage manipulator, responsible for the onset of inflammation cascade. Under unstimulated conditions, NF- $\kappa \mathrm{B}$ inhibitory protein, I $\mathrm{B}$, firmly forms a complex with NF- $\mathrm{BB}$ heterodimer, subunits $\mathrm{p} 50$ and $\mathrm{p} 65$, in cytoplasm. However, once stimulated by inflammatory stimuli, for example, reactive oxygen species (ROS), nitric oxide (NO), or various inflammatory cytokines, the upstream IкB kinase (IKK), is therefore triggered, leading to the phosphorylation of I $\mathrm{B}$ and its subsequent polyubiquitination as well as proteolytic degradation. In this way, NF- $\kappa \mathrm{B}$ pathway is activated by releasing NF- $\mathrm{BB}$ from $\mathrm{I} \kappa \mathrm{B}$ and then translocating into nucleus (Baldwin Jr et al., 1996).

After entering nuclear regions, the free $\mathrm{NF}-\kappa \mathrm{B}$ subunits will specifically bind to promoter DNA sequences and enable the transcription of a series of proinflammatory genes, including inducible nitric oxide synthase (iNOS), tumor necrosis factor- $\alpha$ (TNF- $\alpha$ ), interleukin-1 $\beta$ (IL-1 $\beta)$, cyclooxygenase $2(\mathrm{COX}-2)$ and interleukin-6 (IL-6) (Shin et al., 2012). Among all these downstream targets, TNF- $\alpha$ may be regarded as the most fundamental one for its inducing capacity on activation and accumulation of leukocytes (Li et al., 2018), as well as further initiation of inflammatory cascade by stimulating IL- 6 and IL-1 $\beta$ generation (He et al., 2016). The resulting IL- 6 has proven to be crucial in mediating the release of neutrophils and vascular active substances, which may cause serious tissue impairment (Shin et al., 2015; Lee et al., 2010). Similarly, IL-1 $\beta$ also plays an essential role in the occurrence of visceral organs injuries. Another important mediator, iNOS, is common in both cases of chronic and acute inflammations, and is notable for its stimulating effect on the short-lived effector molecule, NO. By generating reactive oxidant peroxynitrite, NO may ultimately jeopardize splanchnic (Arab et al., 2016; Li et al., 2018) tissue integrity, and induce inflammation-related carcinogenesis and angiogenesis. Similar to iNOS, COX-2 is another inducible proinflammatory enzyme upregulated by activated NF- $\mathrm{KB}$, specifically responsible for the biosynthesis of prostaglandins (PGs) from arachidonic acid (Funaro et al., 2016; Kanaoka et al., 2007). Both COX-2 and PGs, particularly prostaglandin $\mathrm{E}_{2}\left(\mathrm{PGE}_{2}\right)$, a lipid mediator, play pivotal roles in inflammation, tumorigenesis (Ma et al., 2014) and immune system damage. However, opposite to iNOS, the mediator heme oxygenase $1(\mathrm{HO}-1)$ can negatively regulate the $\mathrm{NO}$ generation by its degradation product, carbon monoxide (CO), suggesting the protective role of $\mathrm{HO}-1$ in inflammatory responses by upregulating expression of anti-inflammatory cytokines, such as IL-10 and IL-1RA (interlukin 1 receptor antagonist) (Otterbein et al., 2003).

The above-mentioned mediators or cytokines are all extensively involved in the NF- $\mathrm{kB}$ pathway and can serve as the downstream therapeutic targets for suppressing the development of inflammation. Furthermore, the modulation of their upstream kinases is also of vital importance. It has been well-recognized that the two signaling pathways, PI3K/Akt (phosphoinositide 3-kinase/protein kinase B) and MAPKs (mitogen-activated protein kinases) with three subclasses, JNK (c-Jun N-terminal kinase), p38 and ERK $1 / 2$, are implicated in the translocation of NF- $\mathrm{\kappa B}$ into nucleus ( $\mathrm{Li}$ et al., 2018). Once activated, not only the translocation factor NF$\kappa \mathrm{B}$ is affected, but also some proinflammatory genes, for example, 


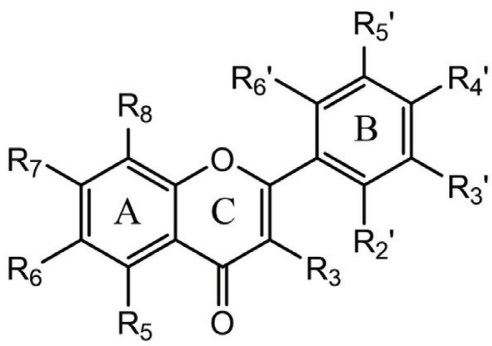

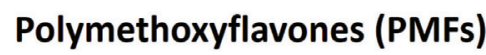

$$
\left(\begin{array}{c}
\mathrm{R}_{3} \text { to } \mathrm{R}_{8} \text { and } \mathrm{R}_{2^{\prime}} \text { to } \mathrm{R}_{6^{\prime}}=\mathrm{H} \text { or } \mathrm{OH} \text { or } \mathrm{OMe} \\
2<=\text { Number of }-\mathrm{OMe}<=7
\end{array}\right)
$$

Figure 2. General chemical structures of polymethoxyflavones.

IL-6 and TNF- $\alpha$, can be directly regulated (Dean et al., 1999; Yasunaga et al., 2016) by these pathways. Evidence shows that apart from those previously discussed kinases, AMPK (5'-adenosine monophosphate-activated protein kinase) is also related to the NF$\kappa \mathrm{B}$-induced inflammatory responses as well as Sirtuin 1 (SIRT1) generation, which facilitates the suppression of NF- $\mathrm{KB}$ (Lee et al., 2016). Thus, phosphorylation of these kinases is also a research focus for NF- $\mathrm{KB}$ pathway blockage.

\subsection{Chemistry of citrus peel PMFs}

As shown in Figure 2, the basic chemical skeleton of PMFs is commonly known as 2-phenylbenzo- $\gamma$-pyrone which is composed of $\mathrm{A}$ and $\mathrm{B}$ phenyl rings attached to the heterocyclic pyrone $\mathrm{C}$ ring that specifically possesses a C-C double bond at the C-2 and C-3 position and bearing at least two methoxy groups (Spencer and Crozier, 2012). Apart from those methoxy groups, the hydrogens on phenyl and pyrone rings of PMFs can also be substituted by hydroxy groups, leading to a large number of PMF family members.

The two most abundant PMFs with prominent bioactivities that exist in citrus peels are nobiletin and tangeretin, commonly detected in Citrus genus, specifically, mandarin oranges (Citrus reticulate) and sweet oranges (Citrus sinensis) (Sergeev et al., 2009). It is intriguing to find out that the discrepancy among citrus species and the degree of citrus fruit ripeness make tremendous difference to the content and variety of PMFs inside pericarp. Some specific types of citrus fruits growing abundantly in Asia, in particular, south China, Japan and Korea, are responsible for the diversity of citrus PMFs. For example, sudachitin is mainly found in Citrus sudachi, which is native to Tokushima Prefecture in Japan (Yuasa et al., 2012), while sinensetin can mostly be extracted from Citrus sunki Hort. ex Tanaka, originated in Jeju Island in Korea. Although the majority of the ten selected citrus PMFs discussed in this contribution are readily separated from various citrus species, as listed in Table 1. Nevertheless, two of which, namely pentamethylquercetin and 5-hydroxy-3,7,3',4'-tetramethoxyflavone, are more enriched in the rhizome of Kaempferia parviflora Wall. ex Baker, a well-known medicinal plant in Thailand which normally referred to as Thai ginseng (Sae-wong et al., 2009). However, it was not until 2015 that the two PMFs mentioned above are eventually discovered from the uninvestigated mandarin species, Miaray Mandarin (Citrus miaray), thus confirming their role as citrus PMFs (Uckoo et al., 2015). Pentamethylquercetin is particularly exceptional for its distinctive source in functional studies. Instead of being isolated from citrus peels, pentamethylquercetin can be easily synthesized from quercetin (Chen et al., 2011). Moreover, tetramethyl-Oscutellarin is also a newly identified citrus PMF which specifically exists in the pericarp of immature Shiranuhi fruit, revealing that the ripeness of citrus fruit may largely change the PMF constituent inside the peel part (Hyun et al., 2017).

In addition to substituting the hydrogen atoms on $\mathrm{A}, \mathrm{B}$ and $\mathrm{C}$ rings of flavone skeleton, the hydroxy groups may also replace the methoxy groups to form the hydroxylated PMFs. The major citrus hydroxylated PMFs identified thus far are 5-demethyl PMFs, commonly generated in aged citrus pericarp as well as citrus fruits undergoing prolonged storage, formed through autohydrolysis of their PMF counterparts (Lai et al., 2015). It is surprisingly to find out that 5-demethyl PMFs may possess superior inhibitory effect on inflammation-induced tumorigenesis than their parent polymethoxylated PMFs (Ma et al., 2014), probably resulting from the better permeability and membrane penetrating activity of the mono- or di-hydroxylated PMFs, leading to their better absorption rate and bioavailability (Wang, Li and Ho, 2018).

\section{Anti-inflammatory activities of citrus PMFs}

\subsection{Inflammatory bowel diseases}

Inflammatory bowel disease (IBD), involving two major forms of chronic inflammation within gastrointestinal tract-ulcerative colitis and Crohn's disease (Podolsky, 1991), is elevated as a gut dysfunction in prevalence in recent years. By developing ulcers in intestinal linings, IBD further leads to abdominal pain and severe diarrhea, and even possibly deteriorate to life-threatening colon cancer. The pathogenesis of IBD, being intrinsically complex, is a combination of immune, gut microbial, genetic and environmental influences (Xiong et al., 2015). According to accumulative evidences, dietary flavonoids possess prominent suppressing effects on various pro-inflammatory cytokines, especially IBD-related key mediators, which has increasingly attracted the attention of researchers. The underlying anti-IBD mechanisms for specific citrus PMFs, nobiletin and tangeretin, are discussed below.

Various studies have suggested that nobiletin may be an effective candidate for treating inflammatory bowel diseases, such as Crohn's disease. It was indicated that by generating restoration effects on Akt (protein kinase B) phosphorylation, PI3K (phosphoinositide 3-kinase) activation, and downstream NF- $\kappa$ B expression, nobiletin administration could down-regulate the expression of two proteins, COX-2 and iNOS (Xiong et al., 2015). The secretions of mediators, $\mathrm{NO}$ and $\mathrm{PGE}_{2}$, and proinflammatory cytokines, IL-1 $\beta$, IL-6 and TNF- $\alpha$ were dramatically suppressed in colitis rats and Caco-2 cell models. Apart from the afore-mentioned proinflammatory cytokines, nobiletin pretreatment also exhibited dramatic downregulation effect on CCL2 (C-C motif chemokine ligand 2), CCL3, CCL4 and CXCL8 (C-X-C motif chemokine ligand 8) in human intestinal mast cells (hiMC), which is a powerful effector cell associated with inflammatory bowel disease. However, nobiletin generated no effect on the expression of IL- $1 \beta$ in IgE (immunoglobulin E) -activated hiMC, while significantly attenuated it in LPS (lipopolysaccharide) -induced hiMC, indicating the suppressing effect of nobiletin may be inducer-dependent (Hagenlocher et al., 2017). Later, the same research group further investigated the impact of nobiletin on in vivo IL-10 $0^{-/-}$mice model. Evidence showed that nobiletin treated mice exhibit significant reduction in colitis clinical symptoms, mast cell numbers, mast cell degranulation and cellular infiltration (Hagenlocher et al., 2018), which may provide very useful reference for clinical application of nobiletin. 
Table 1. The chemical structures and sources of selected citrus PMFs

\begin{tabular}{|c|c|c|c|}
\hline Chemical name & Chemical Structure & Source & Reference \\
\hline Nobiletin & & $\begin{array}{l}\text { sweet orange (Citrus sinensis), } \\
\text { tangerine (Citrus tangerina), } \\
\text { bitter orange (Citrus aurantium) }\end{array}$ & Li et al., 2014 \\
\hline Tangeretin & & $\begin{array}{l}\text { Citrus unshiu, Citrus depressa } \\
\text { (Rutaceae), Citrus reticulata }\end{array}$ & $\begin{array}{l}\text { Eun et al., 2017; } \\
\text { Jang et al., } 2013\end{array}$ \\
\hline 5-Demethyltangeretin & & $\begin{array}{l}\text { sweet orange (Citrus sinensis), } \\
\text { Scrophulariaceae (Limnophila } \\
\text { geoffrayi), Limnophila } \\
\text { aromatic, genus Oscimum }\end{array}$ & Li et al., 2006 \\
\hline $3,5,6,7,8,3^{\prime}, 4^{\prime}$-Heptamethoxyflavone & & $\begin{array}{l}\text { Citrus genus, especially abundant } \\
\text { in Citrus kawachiensis }\end{array}$ & Kou et al., 2013 \\
\hline $\begin{array}{l}\text { 5-Hydroxy-3,6,7,8, } 3^{\prime}, 4^{\prime}- \\
\text { hexamethoxyflavone }\end{array}$ & & $\begin{array}{l}\text { sweet orange (Citrus } \\
\text { sinensis), Hizikia fusiforme }\end{array}$ & $\begin{array}{l}\text { Kang et al., 2013; } \\
\text { Lai et al., } 2007\end{array}$ \\
\hline Sinensetin & & $\begin{array}{l}\text { Citrus sunki Hort. ex Tanaka, } \\
\text { Orthosiphon stamineus }\end{array}$ & $\begin{array}{l}\text { Ko et al., 2010; } \\
\text { Kang et al., 2013; } \\
\text { Laavola et al., } 2012\end{array}$ \\
\hline Pentamethylquercetin & & $\begin{array}{l}\text { Miaray Mandarin (Citrus miaray), } \\
\text { Kaempferia parviflora Wall. ex } \\
\text { Baker rhizomes, sea buckthorn } \\
\text { (Hippophae rhamnoides) }\end{array}$ & $\begin{array}{l}\text { Sae-wong et al., 2011; } \\
\text { Uckoo et al., 2015; } \\
\text { Wang et al., } 2011\end{array}$ \\
\hline 5-Hydroxy-3,7,3',4'-tetramethoxyflavone & & $\begin{array}{l}\text { Miaray Mandarin (Citrus } \\
\text { miaray), Kaempferia parviflora } \\
\text { Wall. ex Baker rhizomes }\end{array}$ & $\begin{array}{l}\text { Uckoo et al., 2015; } \\
\text { Tewtrakul and } \\
\text { Subhadhirasakul, } 2008\end{array}$ \\
\hline Sudachitin & & Citrus sudachi & Yuasa et al., 2012 \\
\hline
\end{tabular}


Table 1. The chemical structures and sources of selected citrus PMFs - (continued)

\begin{tabular}{llll}
\hline Chemical name & Chemical Structure & Source & Reference \\
\hline Tetramethyl-O-scutellarin & Hyun et al., 2017 \\
\hline
\end{tabular}

Another interesting research published recently investigated the synergistic effect of nobiletin and atorvastatin (ATST), a blood cholesterol-lowering drug, on color cancer-induced inflammation both in cell cultures and in a rat model. Nobiletin and ATST synergistically exerted a stronger suppressing effect on NO overproduction in vitro, and in in vivo condition, the synergistic effect of the two compounds also better mitigated the generation of IL-1 $\beta$, IL- 6 and TNF- $\alpha$ in mRNA level, as well as the expression of COX-2 and MMP-9 (matrix metalloproteinases-9) in protein level (Wu et al., 2017). These findings verified that the inflammatory effect of nobiletin may broadly count on the suppression of PI3K/Akt pathway as well as the activated NF- $\mathrm{kB}$, which serves as crucial hub of the whole inflammatory pathway.

The anti-inflammatory effect of tangeretin on LPS-induced bone marrow cells and colitic mice was respectively examined (Eun et al., 2017). In both models, tangeretin significantly suppressed IL-12 and TNF- $\alpha$ expression by regulating NF- $\kappa$ B, as well as the iNOS and COX-2 generation. However, IL-10 level was not affected. Besides, the NF- $\kappa \mathrm{B}$ translocation and activation were inhibited by the downregulated phosphorylation of IKK $\alpha / \beta$ and $\mathrm{I} \kappa \mathrm{B} \alpha$, and the activation of three AMPK subclasses, p38, ERK and JNK, was also counteracted.

Tangeretin exhibited an overall weaker ability than nobiletin in treating inflammation in human intestinal mast cells. Although tangeretin could down-regulate IL-1 $\beta$, CCL2, CCL3, CCL4 and CXCL8 expressions, nobiletin was found possessing better adverse effect on the last two cytokines. It is noteworthy that the IL-1 $\beta$ level was only dramatically suppressed by tangeretin, not by nobiletin (Hagenlocher et al., 2017), indicating the specific inhibitory effects of tangeretin in inflammatory responses.

\subsection{Neuroinflammation}

Neuroinflammation is usually stimulated by the activation of microglial cells, the resident immune cells inside the brain, resulting from environmental stimuli or tissue injuries. After stimulation, the microglial cells can subsequently generate various proinflammatory mediators, particularly NO, MMPs, IL- $1 \beta$ and TNF- $\alpha$, all of which are implicated in the death of neuron (Ho and Kuo, 2014). Although the activated microglial cells also facilitate the production of anti-inflammatory cytokines, the prolonged activation time may counteract the positive effects that those mediators bring about (Lee et al., 2016), further causing homeostatic imbalance in the brain and the consequent inflammatory responses.

Regarding the anti-neuroinflammatory effect of nobiletin, the secretion of iNOS, TNF- $\alpha$, IL- 6 and IL- $1 \beta$ was reported to be dramatically terminated both at protein and mRNA levels in microglia cells, implying that the down-regulation of gene expression may contribute to the attenuating effect of nobiletin on proinflammatory cytokine production (Ho and Kuo, 2014). Besides, treating rats with nobiletin intraperitoneally also exerted anti-neuroinflammatory effect on isoflurane-induced cognitive impairment. Despite the administration being only within a short span of 3 days, nobiletin still dramatically decreased the TNF- $\alpha$, IL- $1 \beta$, IL- 6 and NF- $\kappa B$ expression levels (Bi et al., 2016).

Meanwhile, a specific kind of neuroinflammation, ischemic stroke, activated by ischemia, was also investigated on pMCAO (permanent middle cerebral artery occlusion) rat models. Rats intraperitoneally receiving nobiletin exhibited a significantly reduced NF- $\mathrm{KB}$ expression in nucleus, and certainly, enhanced

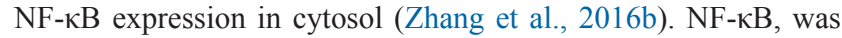
further verified to play an irreplaceable role in neuroinflammation pathogenesis (Harari and Liao, 2010). However, the definite NF$\kappa \mathrm{B}$ pathway that is responsible for the inflammation-induced brain injury requires further confirmation.

Detailed underlying mechanistic study aiming at investigating the anti-neuroinflammatory effect of tangeretin was carried out on LPS-activated microglial cells. Apart from the well-studied influence on IL- 6 , IL-1 $\beta$, TNF- $\alpha$, iNOS and NO, tangeretin also mediated the anti-inflammatory protein TIMP-2 (tissue inhibitor of metalloproteinases-2) as well as pro-inflammatory target proteins, MMP-3 and MMP-8, instead of MMP-9, while the balance maintained between them is imperative for neuron health. In addition to the phosphorylation of MAPKs, Akt and AMPK, SIRT1 expression and the closely related NF- $\kappa B$ activation were all modulated by tangeretin, suggesting the definite role of tangeretin in neuroinflammation and could be the regulator of AMPK/SIRT1/NF- $\kappa B$ signaling pathway (Lee et al., 2016).

However, tangeretin when compared with nobeltin exhibited a much weaker capacity in mitigating the expression of all five antiinflammatory mediators mentioned above in LPS-induced microglial system (Ho and Kuo, 2014), proving that the extra methoxy group on the $\mathrm{B}$ ring indeed leads to better functionalities.

Moreover, the biofunctional role of $3,5,6,7,8,3^{\prime}, 4^{\prime}$-heptamethoxyflavone (HMF) against ischemia-induced brain inflammation was indicated by Okuyama et al. (2014) from pathological point of view. After treatment, HMF could effectively restore the activated ameboid-like microglia to their original ramified form and significantly restrain the IBA1 (ionized calcium binding adaptor molecule 1) generation, which is the signal of nerve injury. Later, the underlying molecular mechanism was further interpreted by stating that HMF reversed the neuroinflammation via blockage of IL-1 $\beta$ expression at gene level. However, HMF barely exhibited any inhibitory effect on other possible inflammatory targets, namely, COX-2, TNF- $\alpha$ and iNOS, which needs further investigation (Okuyama et al., 2015). In the case of 5-hydroxy-3,6,7,8,3',4'hexamethoxyflavone (5HHMF)-treated LPS-induced microglia cells, it was suggested that by first blocking the I $\mathrm{B}$ phosphorylation along with subsequent NF- $\kappa \mathrm{B}$ translocation and then inducing the Nrf2 (nuclear factor erythroid-2 related factor) -related HO-1 generation, 5HHMF could instead suppress iNOS expression and subsequent NO production in neuro-system (Kang et al., 2013). This reveals that the substitution of methoxy group at the C-5 position by a hydroxy group may bring change to the underlying antiinflammatory mechanisms of PMFs. 


\subsection{Organ inflammation}

The pathogenesis of inflammation-induced end-organ impairment can be partly treated as autoimmune disorder, which can be classified as two types: (1) acute inflammation-induced toxicity such as cisplatin caused acute injury; and (2) pathogen induced inflammation causing poor blood circulation along with consequent chronic organ dysfunction. The citrus PMFs-related anti-organ inflammation studies are mainly focused on four of the end-organs, namely gastric, liver, kidney and lung, which are separately discussed later.

It was evidenced that nobiletin exhibits ameliorating effects on numerous acute visceral injuries (He et al., 2016; Li et al., 2017; Li et al., 2018; Malik et al., 2015). Cisplatin is a first line anticancer drug, exhibiting severe toxicity towards kidney and liver partially via stimulating inflammation responses. In addressing cisplatin-induced renal-injury, Malik et al. (2015) discovered that pretreatment with as low as $5 \mathrm{mg} / \mathrm{kg}$ of nobiletin could mitigate the elevated TNF- $\alpha$ level nearly down to the normal level. Regarding the histopathological effect of nobiletin on damaged kidney, nobiletin at the same dosage also remarkably exerted anti-interstitial inflammatory action at tissue level (Malik et al., 2015). Thereby, this study may reveal that nobiletin can be co-administrated with cisplatin at a relatively low dosage in the hope of eliminating malignant tumors. Nobiletin was also proven to possess ameliorative influence on LPS/GalN (D-galactosamine) — induced acute hepatic injury in vivo (He et al., 2016). Administration of nobiletin intraperitoneally prior to liver damage was detected to significantly reverse the enhanced levels of inflammatory mediator TNF- $\alpha$ and its correlative cytokines, IL- 6 and IL-1 $\beta$, as well as upstream proteins, iNOS and COX-2, in a dosage-dependent manner. Besides, the degradation and phosphorylation of $I \kappa \mathrm{B} \alpha$ and the subsequent translocation of NF- $\mathrm{KB}$ p 65 were mitigated by nobiletin, while the expression of Nrf2, which can remit the NF- $\mathrm{kB}$-related inflammation (Onda et al., 2013), was adversely affected, suggesting that the suppressing effect of nobiletin on NF- $\kappa \mathrm{B}$ may be attributed to the Nrf2 activation. Moreover, nobiletin intuitively showed attenuating effect on hepatic inflammation by reversing the liver pathological injuries involving portal inflammation and infiltration of inflammatory cells. Similar results were indicated later in the study of pretreating LPS-induced pulmonary injury with nobiletin. Apart from the mechanisms discussed above, NO generation was reported to be reduced exclusively in lung tissue and bronchoalveolar lavage fluid (BALF) of rats ( $\mathrm{Li}$ et al., 2018). However, in the case of ethanol-induced gastric injury, the expression of phosphorylated-p38, JNK and ERK1/2 (extracellular-signal-regulated kinase $1 / 2$ ), the three subgroups belonging to MAPK family were also verifiably restrained, indicating direct restriction on their regulation of downstream mediators (Li et al., 2017). These researches provided evidences that nobiletin may mitigate various acute tissue injuries prospectively. However, the influence of nobiletin on inflammation mentioned already still needs further investigation and evaluation.

In treating rats with cisplatin-induced acute liver injury, tangeretin mainly plays its role in suppressing TNF- $\alpha$ levels, restoring IL-10 content as well as inhibiting the phosphorylation of three MAPK family proteins, p38, JNK and ERK1/2. During this process, tangeretin, at the same level, exhibited a similar degree of inhibition as silymarin, a commercial liver-protective agent (Omar et al., 2016). It was also indicated that tangeretin could cure nephrectomy-induced chronic kidney injury and the associated memory impairment, which are closely correlated with inflammatory responses, by reducing the levels of inflammatory mediators, NO, IL- $1 \beta$, IL- 6 and TNF- $\alpha$ through regulating I $\mathrm{B} \alpha$, IKK $\alpha$, IKK $\beta$ and iNOS phosphorylation as well as NF- $\kappa \mathrm{B}$ p65 expression. In brief, tangeretin can specifically act on NF- $\kappa \mathrm{B} / \mathrm{TNF}-\alpha / \mathrm{iNOS}$ signaling pathways when dealing with kidney-related injuries (Wu et al., 2018).

Similar results were again found when tangeretin treatment was carried out in both cases of cisplatin-induced acute renal deficit (Arab et al., 2016) and RSV infection-stimulated lung inflammation (Xu et al., 2015). This demonstrates the powerful effect of tangeretin on inflammatory response attenuation through targeting NF- $\kappa$ B pathways.

\subsection{LPS-induced inflammation}

Being the main component of the outer membrane of Gram-negative bacteria, LPS is capable of evoking inflammatory responses in macrophages, thus playing pivotal roles in immune system. This leads to the secretion of various pro-inflammatory mediators (Zhang and Ghosh, 2000). Therefore, LPS-induced inflammation has been employed as a practical model in evaluating anti-inflammatory capacity of novel agents by detecting their down-regulatory effects on pro-inflammatory cytokines.

Interestingly, by synergizing with luteolin, a dietary flavonoid, tangeretin even showed a superior ability in suppressing the proinflammatory mediators, IL-6, IL- $1 \beta, \mathrm{PGE}_{2}$ and $\mathrm{NO}$, meanwhile reducing the expression of pro-inflammatory enzymes, COX-2 and iNOS, in LPS-induced macrophages (Funaro et al., 2016).

A study conducted by Manthey et al (1999), reported that $3,5,6,7,8,3^{\prime}, 4^{\prime}$-heptamethoxyflavone (HMF) not only significantly suppressed the TNF- $\alpha$ response in LPS-treated mice, but also rendered adverse effects on MIP- $1 \alpha$ (macrophage inflammatory protein $1 \alpha$ ) and IL-10 expression, although no impact was found towards cytokines IL-6, IL- 8 and IL- $1 \beta$. An interesting finding was also highlighted by these researchers that among a great part of PMFs inside citrus peel, HMF exhibited the highest capacity in restraining TNF- $\alpha$ secretion. Thus, this may provide guidance toward using HMF in treating specific inflammatory diseases.

Shin et al. (2012) reported that sinensetin might prospectively be employed in inflammation treatment due to its inhibitory effects on $\mathrm{NF}-\kappa \mathrm{B}$ pathway by fundamentally stabilizing I $\mathrm{I} \mathrm{B}-\alpha$ and reducing p65 level in the nucleus. In this way, the NF- $\mathrm{KB}$-activated expression of inflammatory mediators, TNF- $\alpha$, COX- 2 , iNOS, NO, IL- $1 \beta$ and IL-6 was tremendously delayed. However, another study indicated that translocation of NF- $\mathrm{kB}$ p 65 was not regulated by sinensetin, while STAT1 $\alpha$ (signal transducer and activator of transcription $1 \alpha)$ activation was instead mediated. Being the central pro-inflammatory transcription factor like NF- $\mathrm{B}$, STAT $1 \alpha$ mediation further led to the reduction in COX-2 and iNOS expression as well as generation of TNF- $\alpha, \mathrm{PGE}_{2}$ and NO. It is worth noting that sinensetin exhibited superior effect on COX-2, TNF- $\alpha$ and $\mathrm{PGE}_{2}$ regulation, with $\mathrm{IC}_{50}$ value so low as $2.7 \mu \mathrm{M}$ (Laavola et al., 2012).

The anti-inflammatory effect of pentamethylquercetin requires further study probably due to its poor performance on LPS-induced $\mathrm{NO}$ production in murine peritoneal macrophages $\left(\mathrm{IC}_{50}=\right.$ $26 \mu \mathrm{M}$ ) (Matsuda et al., 2003) and RAW 267.4 cells $\left(\mathrm{IC}_{50}=258\right.$ $\mu \mathrm{M})$ (Sae-wong et al., 2011), suggesting that the repelling activity of pentamethylquercetin against inflammation may deserve further investigation, at least, for other aspects.

Three studies carried out nearly a decade ago briefly interpreted the molecular mechanism behind the bioactivity of 5-hydroxy$3,7,3^{\prime}, 4^{\prime}$-tetramethoxyflavone. It was believed that the anti-inflammatory property was due to the suppressing effect on iNOS mRNA expression (Sae-wong et al., 2009) as well as the accompanying $\mathrm{PGE}_{2}$ and NO secretion $\left(\mathrm{IC}_{50} \sim 16 \mu \mathrm{M}\right)$ (Tewtrakul and Subhadhirasakul, 2008; Tewtrakul et al., 2009), although not as potent as other 


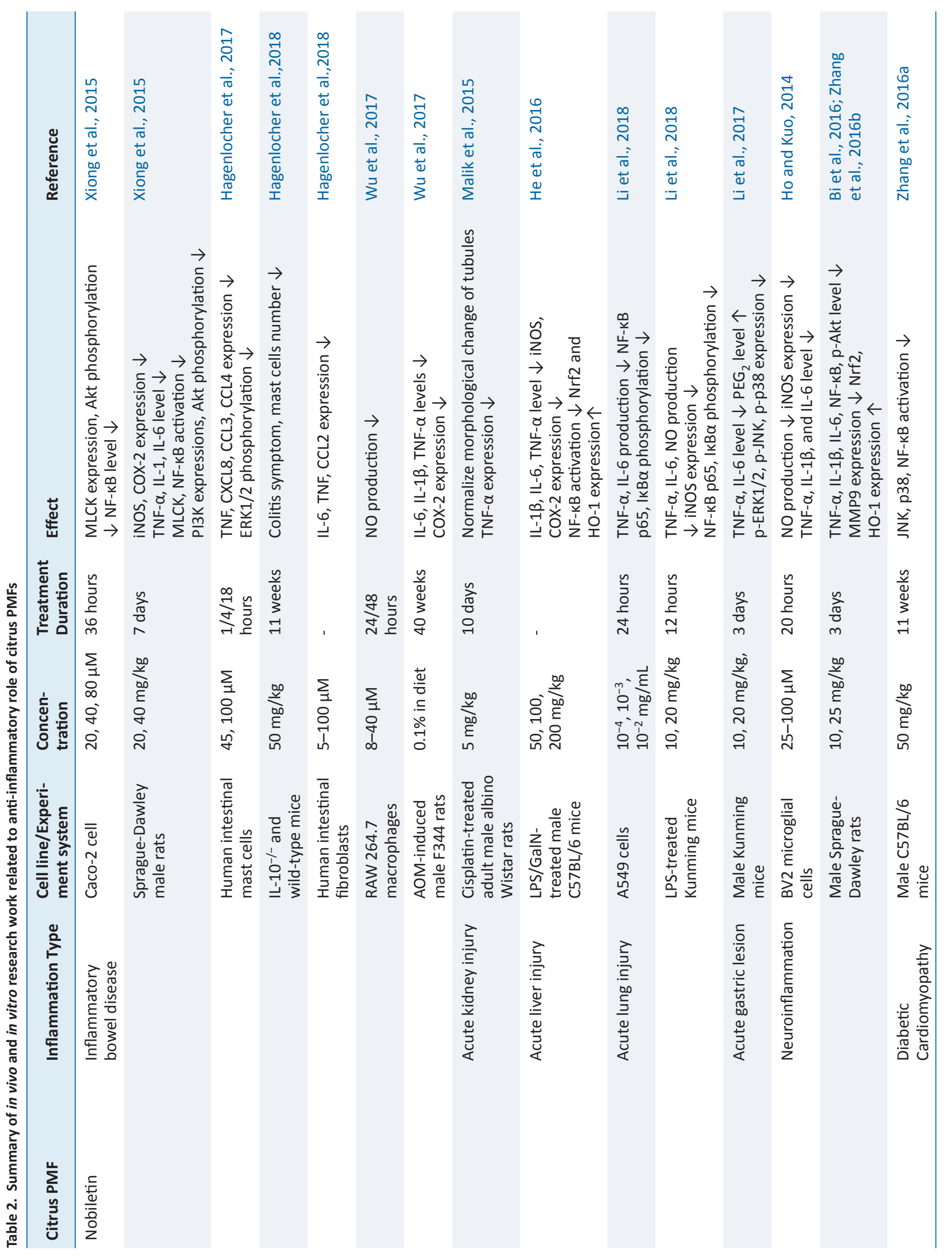




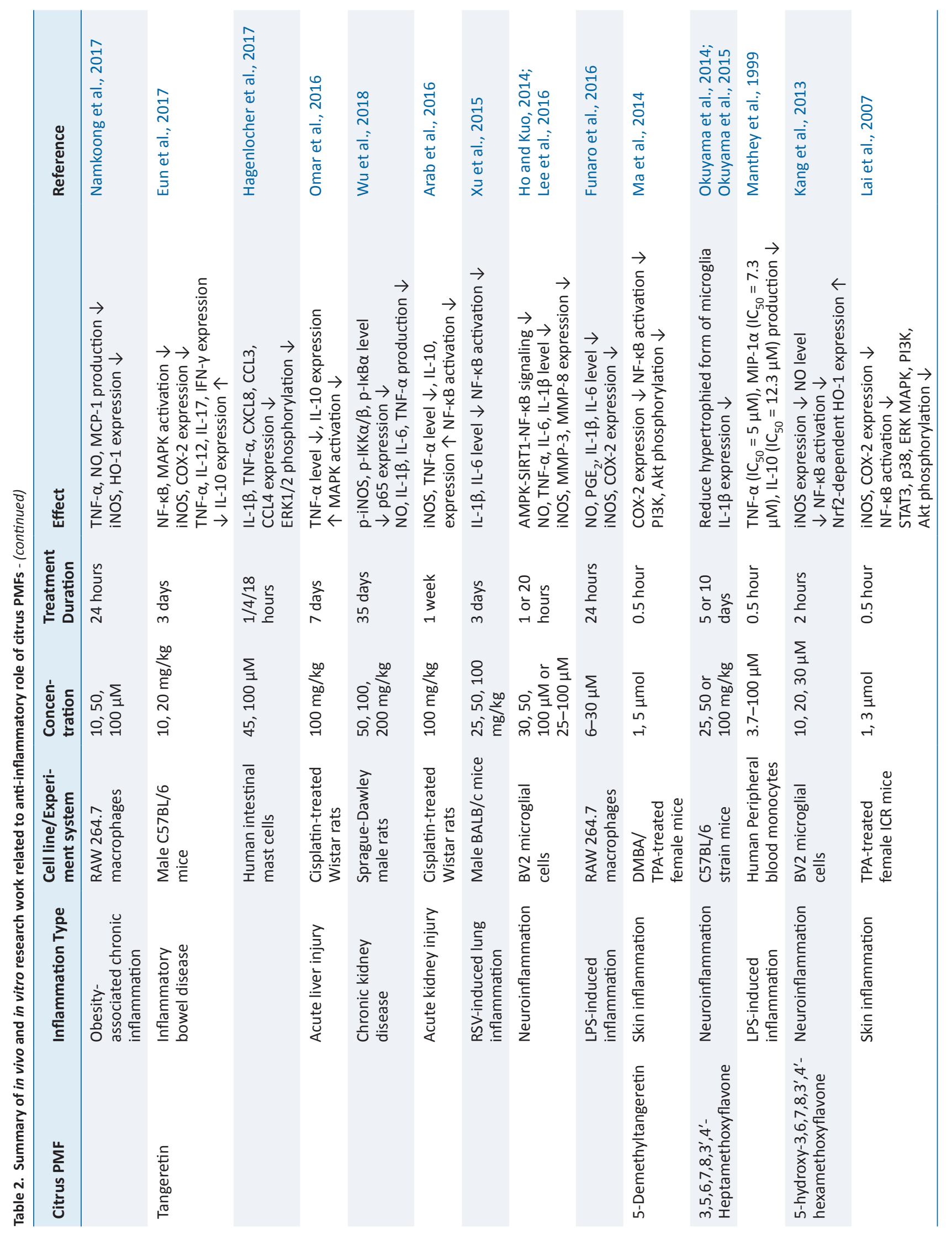


citrus PMFs. However, it was also determined that 5-hydroxy$3,7,3^{\prime}, 4^{\prime}$-tetramethoxyflavone treatment made no difference to the TNF- $\alpha$ and COX-2 levels in LPS-activated macrophages. Impressively, sudachitin showed much better effects than nobiletin in mitigating TNF- $\alpha$ and NO production as well as the upstream iNOS expression in LPS-stimulated macrophages (Yuasa et al., 2012), indicating that demethylation at certain position may contribute to enhanced anti-inflammatory activity.

Furthermore, evidence shows that tetramethyl- $O$-scutellarin reveals moderate reducing effect on $\mathrm{NO}$ generation within macrophages $\left(\mathrm{IC}_{50}=57.4 \mu \mathrm{M}\right)$. In addition, the mediator $\mathrm{PGE}_{2}$ together with several relevant cytokines, namely, IL-1 $\beta$, IL- 6 and TNF- $\alpha$, were also inhibited to varying degrees after treatment, indicating tetramethyl- $O$-scutellarin indeed possessed certain anti-inflammatory activities (Hyun et al., 2017).

\subsection{Metabolic syndrome-induced inflammation}

The chronic cardiac inflammation is of vital importance in the development of diabetic cardiomyopathy (Aragno et al., 2006) and the two downstream signaling pathways, namely MAPK and NF- $\kappa B$, are implicated in the inflammatory response to cardiac dysfunction. Zhang et al. (2016a) studied the diabetic cardiomyopathy induced by streptozotocin in vivo and concluded that nobiletin treatment can ameliorate the levels of two MAPK subfamilies, JNK and p38, as well as NF- $\kappa \mathrm{B}$ in diabetic mice, probably by blocking the activation of p-JNK, p-p38, p-IкB $\alpha$ and p-p65. In addition, the mRNA expression of IL- 6 and TNF- $\alpha$, which indicates aggravating cardiac impairment, was also downregulated by nobiletin.

Chronic inflammation is closely associated with obesity, lying in the infiltration of macrophage into adipose tissue. A recent research explored the plausible inflammatory response based on the crosstalk between macrophages and differentiated adipocytes, as well as the anti-inflammatory effect of nobiletin, and suggested that the elevated secretion of TNF- $\alpha$ and NO, along with the expression of iNOS induced by interaction between macrophages and adipocytes are all weakened by nobiletin treatment. Moreover, essential induction of HO-1, a beneficial enzyme against chronic inflammation, was detected as well. By blocking NO production, HO-1 may serve as the therapeutic target within inflammatory response and facilitate the suppression of obesity-induced inflammation (Namkoong et al., 2017).

\subsection{Skin inflammation}

Occurrence of skin inflammation takes place in people's daily life, simply due to internal or external irritations, and subsequently resulting in burning or itching of skin and inflammation or even malicious tumorigenesis over a long period of time.

The potential molecular mechanism of 5-demethyltangeretin in suppressing inflammation-induced skin carcinogenesis was investigated. Briefly, the modulation of PI3K/Akt signaling pathway via 5-demethyltangeretin started with the mitigation of upstream Akt and PI3K phosphorylation, then the consequent obstruction of $\mathrm{I} \kappa \mathrm{B} \alpha$ degradation as well as NF- $\mathrm{B}$ translocation, and further led to the suppression of COX-2 expression (Ma et al., 2014). In this study, 5-demethyltangeretin was demonstrated to exert a better anti-inflammatory potential than its parent PMF. However, more efforts are required to investigate the antiphlogistic role of 5-demethyltangeretin and 5-demethylnobiletin, as well as the comparison between their bioactivities. In addition, 5-hydroxy$3,6,7,8,3^{\prime}, 4^{\prime}$-hexamethoxyflavone (5HHMF) was also proven to 
generate anti-inflammatory influences on I $\mathrm{B}-\alpha$ degradation, NF$\kappa \mathrm{B}$ activation, and TPA-induced $\mathrm{NNOS}$ and COX-2 expression in inflamed mouse skin. Moreover, the activations of three upstream signals, p38 MAPK, ERK1/2 and PI3K/Akt were all detected to be interrupted, plausibly owing to the masking effect of 5HHMF towards their phosphorylation process (Lai et al., 2007). As a result, both the MAPK and PI3K/Akt/IKK may be responsible for the modulation of NF- $\mathrm{KB}$ in the case of skin inflammation.

\section{Conclusions and prospects}

Numerous in vivo and in vitro studies have verified the prospective anti-inflammatory roles of various citrus PMFs in the prevention and treatment of functional and immune disabilities (Table 2). Citrus peel PMFs are mainly exerting inhibitory effects on PI3K/ Akt/IKK and AMPK/SIRT1/NF- $\mathrm{BB}$ pathways in inflammatory responses. However, research targets for the majority of the available investigations are focused on the most abundant PMFs present in citrus peels, namely nobiletin, tangeretin and 3,5,6,7,8,3', $4^{\prime}$-heptamethoxyflavone. It was demonstrated that pentamethylquercetin from Citrus miaray (Uckoo et al., 2015) can be readily obtained in high purity via chemical synthesis. However, direct bulk extraction, isolation and purification of PMFs and further exploration of PMF biological activities deserve special attention. Furthermore, 5-demethyltangeretin exerted a more potent activity towards inflammation-related disease compared with its parent polymethoxylated compound, suggesting the superior outcome of substitution of methoxy groups with hydroxy groups. Thus, more efforts should focus on exploring the functional activities of other mono- or di-hydroxylated citrus PMFs. In addition, there still remains unexplored mechanism-implicated problems to be investigated within the reported studies and beyond. For example, the post-treatment effects of citrus PMFs on numerous inflammations, as well as the definite $\mathrm{NF}-\mathrm{\kappa B}$ pathway that is involved in the anti-inflammatory response of citrus PMFs need to be examined in detail. Overcoming these difficulties may pave the way for the application of citrus PMFs in clinical therapy towards numerous inflammatory complications.

\section{Acknowledgments}

We sincerely thank Jian Gao (Institute of Plant Physiology and Ecology, CAS) for his help on figure modification.

\section{Conflict of interest}

There are no conflicts of interest to declare.

\section{References}

Arab, H.H., Mohamed, W.R., Barakat, B.M., and Arafa, E.S.A. (2016). Tangeretin attenuates cisplatin-induced renal injury in rats: Impact on the inflammatory cascade and oxidative perturbations. Chem. Biol. Interact. 258: 205-213.

Aragno, M., Mastrocola, R., Medana, C., Catalano, M.G., Vercellinatto, I., Danni, O., and Boccuzzi, G. (2006). Oxidative stress-dependent impairment of cardiac-specific transcription factors in experimental diabetes. Endocrinology. 147(12): 5967-5974.

Baldwin Jr, A.S. (1996). The NF-kB and IKB proteins: new discoveries and insights. Ann. Rev. Immunol. 14(1): 649-683.

$\mathrm{Bi}$, J., Zhang, H., Lu, J., and Lei, W. (2016). Nobiletin ameliorates isoflurane- induced cognitive impairment via antioxidant, anti-inflammatory and anti-apoptotic effects in aging rats. Mol. Med. Rep. 14(6): 5408-5414.

Chen, L., He, T., Han, Y., Sheng, J.Z., Jin, S., and Jin, M.W. (2011). Pentamethylquercetin improves adiponectin expression in differentiated 3T3-L1 cells via a mechanism that implicates PPARY together with TNF- $\alpha$ and IL-6. Molecules 16(7): 5754-5768.

Dean, J.L., Brook, M., Clark, A.R., and Saklatvala, J. (1999). p38 mitogenactivated protein kinase regulates cyclooxygenase-2 mRNA stability and transcription in lipopolysaccharide-treated human monocytes. J. Biol. Chem. 274(1): 264-269.

Eun, S.H., Woo, J.T., and Kim, D.H. (2017). Tangeretin inhibits IL-12 expression and NF-KB activation in dendritic cells and attenuates colitis in mice. Planta Med. 83(06): 527-533.

Funaro, A., Wu, X., Song, M., Zheng, J., Guo, S., Rakariyatham, K., Rodriguez-Estrada, M.T., and Xiao, H. (2016). Enhanced anti-inflammatory activities by the combination of luteolin and tangeretin. J. Food Sci. 81(5): H1320-1327.

Hagenlocher, Y., Feilhauer, K., Schaffer, M., Bischoff, S.C., and Lorentz, A. (2017). Citrus peel polymethoxyflavones nobiletin and tangeretin suppress LPS- and IgE-mediated activation of human intestinal mast cells. Eur. J. Nutr. 56(4): 1609-1620.

Hagenlocher, Y., Gommeringer, S., Held, A., Feilhauer, K., Koninger, J., Bischoff, S.C., and Lorentz, A. (2018). Nobiletin acts anti-inflammatory on murine $\mathrm{IL}-10^{-/}$colitis and human intestinal fibroblasts. Eur. J. Nutr. $1-11$.

Harari, O.A., and Liao, J.K. (2010). NF-KB and innate immunity in ischemic stroke. Ann. N. Y. Acad. Sci. 1207(1): 32-40.

He, Z., Li, X., Chen, H., He, K., Liu, Y., and Gong, J. (2016). Nobiletin attenuates lipopolysaccharide/D-galactosamine-induced liver injury in mice by activating the Nrf2 antioxidant pathway and subsequently inhibiting NF-KB-mediated cytokine production. Mol. Med. Rep. 14(6): 5595-5600.

Ho, S.C., and Kuo, C.T. (2014). Hesperidin, nobiletin, and tangeretin are collectively responsible for the anti-neuroinflammatory capacity of tangerine peel (Citri reticulatae pericarpium). Food Chem. Toxicol. 71: 176-182.

Hyun, J.M., Jo, Y.J., Kim, J.E., An, H.J., Choi, Y.H., Hyun, C.G., and Lee, N.H. (2017). Tetramethyl-O-scutellarin isolated from peels of immature Shiranuhi fruit exhibits anti-inflammatory effects on LPS induced RAW264. 7 cells. Trop. J. Pharm. Res. 16(9): 2197-2205.

Jang, S.E., Ryu, K.R., Park, S.H., Chung, S., Teruya, Y., Han, M.J., Woo, J.T., and Kim, D.H. (2013). Nobiletin and tangeretin ameliorate scratching behavior in mice by inhibiting the action of histamine and the activation of NF-KB, AP-1 and p38. Int. Immunopharmacol. 17(3): 502-507.

Kanaoka, S., Takai, T., and Yoshida, K. (2007). Cyclooxygenase-2 and tumor biology. Adv. Clin. Chem. 43: 59-78.

Kang, C.H., Kim, M.J., Seo, M.J., Choi, Y.H., Jo, W.S., Lee, K.T., Jeong, Y.K., and Kim, G.Y. (2013). 5-Hydroxy-3,6,7,8,3', $4^{\prime}$-hexamethoxyflavone inhibits nitric oxide production in lipopolysaccharide-stimulated BV2 microglia via NF-KB suppression and Nrf-2-dependent heme oxygenase-1 induction. Food Chem. Toxicol. 57: 119-125.

Kang, S.I., Shin, H.S., Ko, H.C., and Kim, S.J. (2013). Effects of sinensetin on lipid metabolism in mature 3T3-L1 adipocytes. Phytother. Res. 27(1): 131-134.

Ko, H.C., Jang, M.G., Kang, C.H., Lee, N.H., Kang, S.I, Lee, S.R., Park, D.B., and Kim, S.J. (2010). Preparation of a polymethoxyflavone-rich fraction (PRF) of Citrus sunki Hort. ex Tanaka and its antiproliferative effects. Food Chem. 123(2): 484-488.

Kou, M.C., Fu, S.H., Yen, J.H., Weng, C.Y., Li, S., Ho, C.T., and Wu, M.J. (2013). Effects of citrus flavonoids, 5-hydroxy-3,6,7,8,3', $4^{\prime}$-hexamethoxyflavone and $3,5,6,7,8,3^{\prime}, 4^{\prime}$-heptamethoxyflavone, on the activities of macrophage scavenger receptors and the hepatic LDL receptor. Food Funct. 4(4): 602-609.

Laavola, M., Nieminen, R., Yam, M.F., Sadikun, A., Asmawi, M.Z., Basir, R., Welling, J., Vapaatalo, H., Korhonen, R., and Moilanen, E. (2012). Flavonoids eupatorin and sinensetin present in Orthosiphon stamineus leaves inhibit inflammatory gene expression and STAT1 activation. Planta Med. 78(08): 779-786.

Lai, C.S., Li, S., Chai, C.Y., Lo, C.Y., Ho, C.T., Wang, Y.J., and Pan, M.H. (2007). Inhibitory effect of citrus 5-hydroxy-3,6,7,8,3', 4'-hexamethoxyflavone on 12-O-tetradecanoylphorbol 13 -acetate-induced skin inflamma- 
tion and tumor promotion in mice. Carcinogenesis 28(12): 25812588.

Lai, C.S., Wu, J.C., Ho, C.T., and Pan, M.H. (2015). Disease chemopreventive effects and molecular mechanisms of hydroxylated polymethoxyflavones. Biofactors 41(5): 301-313.

Lee, Y.S., Cha, B.Y., Saito, K., Yamakawa, H., Choi, S.S., Yamaguchi, K., Yonezawa, T., Teruya, T., Nagai, K., and Woo, J.T. (2010). Nobiletin improves hyperglycemia and insulin resistance in obese diabetic $o b / o b$ mice. Biochem. Pharmacol. 79(11): 1674-1683.

Lee, Y.Y., Lee, E.J., Park, J.S., Jang, S.E., Kim, D.H., and Kim, H.S. (2016). Antiinflammatory and antioxidant mechanism of tangeretin in activated microglia. J. Neuroimmune Pharmacol. 11(2): 294-305.

Li, S., Lo, C.Y., and Ho, C.T. (2006). Hydroxylated polymethoxyflavones and methylated flavonoids in sweet orange (Citrus sinensis) peel. J. Agric. Food Chem. 54(12): 4176-4185.

Li, S., Wang, H., Guo, L., Zhao, H., and Ho, C.T. (2014). Chemistry and bioactivity of nobiletin and its metabolites. J. Funct. Foods 6: 2-10.

Li, W., Wang, X., Zhi, W., Zhang, H., He, Z., Wang, Y., Liu, F., Niu, X., and Zhang, X. (2017). The gastroprotective effect of nobiletin against ethanol-induced acute gastric lesions in mice: impact on oxidative stress and inflammation. Immunopharmacol. Immunotoxicol. 39(6): 354-363.

Li, W., Zhao, R., Wang, X., Liu, F., Zhao, J., Yao, Q., Zhi, W., He, Z., and Niu, X. (2018). Nobiletin-ameliorated lipopolysaccharide-induced inflammation in acute lung injury by suppression of NF-KB pathway in vivo and vitro. Inflammation. 41(3): 996-1007.

Ma, N., Lai, C.S., Chung, C.H., Yang, J.M., Hsu, K.C., Chen, C.Y., Chung, T.S., Li, S., Ho, C.T., and Pan, M.H. (2014). 5-Demethyltangeretin is more potent than tangeretin in inhibiting dimethylbenz (a) anthracene (DMBA)/12-O-tetradecanoylphorbol-13-acetate (TPA)-induced skin tumorigenesis. J. Funct. Foods. 11: 528-537.

Malik, S., Bhatia, J., Suchal, K., Gamad, N., Dinda, A.K., Gupta, Y.K., and Arya, D.S. (2015). Nobiletin ameliorates cisplatin-induced acute kidney injury due to its anti-oxidant, anti-inflammatory and anti-apoptotic effects. Exp. Toxicol. Pathol. 67(7-8): 427-433.

Manthey, J.A., Grohmann, K., Montanari, A., Ash, K., and Manthey, C.L. (1999). Polymethoxylated flavones derived from citrus suppress tumor necrosis factor- $\alpha$ expression by human monocytes. J. Nat. Prod. 62(3): 441-444

Matsuda, H., Wang, T., Managi, H., and Yoshikawa, M. (2003). Structural requirements of flavonoids for inhibition of protein glycation and radical scavenging activities. Bioorg. Med. Chem. 11(24): 5317-5323.

Namkoong, S., Sung, J., Yang, J., Choi, Y., Jeong, H.S., and Lee, J. (2017) Nobiletin attenuates the inflammatory response through heme oxygenase-1 induction in the crosstalk between adipocytes and macrophages. J. Med. Food 20(9): 873-881.

Okuyama, S., Miyoshi, K., Tsumura, Y., Amakura, Y., Yoshimura, M., Yoshida, T., Nakajima, M., and Furukawa, Y. (2015). 3,5,6,7,8,3',4'-heptamethoxyflavone, a citrus polymethoxylated flavone, attenuates inflammation in the mouse hippocampus. Brain Sci. 5(2): 118-129.

Okuyama, S., Morita, M., Miyoshi, K., Nishigawa, Y., Kaji, M., Sawamoto, A., Terugo, T., Toyoda, N., Makihata, N., Amakura, Y., Yoshimura, M., Nakajima, M., and Furukawa, Y. (2014). 3,5,6,7,8,3',4'-Heptamethoxyflavone, a citrus flavonoid, on protection against memory impairment and neuronal cell death in a global cerebral ischemia mouse model. Neurochem. Int. 70: 30-38.

Omar, H.A., Mohamed, W.R., Arab, H.H., and Arafa, E.S.A. (2016). Tangeretin alleviates cisplatin-induced acute hepatic injury in rats: targeting MAPKs and apoptosis. PLoS One 11(3): e0151649.

Onda, K., Horike, N., Suzuki, T., and Hirano, T. (2013). Polymethoxyflavonoids tangeretin and nobiletin increase glucose uptake in murine adipocytes. Phytother. Res. 27(2): 312-316.

Otterbein, L.E., Soares, M.P., Yamashita, K., and Bach, F.H. (2003). Heme oxygenase-1: unleashing the protective properties of heme. Trends Immunol. 24(8): 449-455.

Podolsky, D.K. (1991). Inflammatory bowel disease. N. Engl. J. Med. 325(13): 928-937

Sae-wong, C., Matsuda, H., Tewtrakul, S., Tansakul, P., Nakamura, S., Nomura, Y., and Yoshikawa, M. (2011). Suppressive effects of methoxyflavonoids isolated from Kaempferia parviflora on inducible nitric oxide synthase (iNOS) expression in RAW 264.7 cells. J. Ethnophar- macol. 136(3): 488-495.

Sae-wong, C., Tansakul, P., and Tewtrakul, S. (2009). Anti-inflammatory mechanism of Kaempferia parviflora in murine macrophage cells (RAW 264.7) and in experimental animals. J. Ethnopharmacol. 124(3): 576-580.

Sergeev, I.N., Li, S., Ho, C.T., Rawson, N.E., and Dushenkov, S. (2009). Polymethoxyflavones activate $\mathrm{Ca}^{2+}$-dependent apoptotic targets in adipocytes. J. Agric. Food Chem. 57(13): 5771-5776.

Shin, H.S., Kang, S.I., Yoon, S.A., Ko, H.C., and Kim, S.J. (2012). Sinensetin attenuates LPS-induced inflammation by regulating the protein level of IKB- $\alpha$. Biosci. Biotechnol. Biochem. 76(4): 847-849.

Shin, N.R., Shin, I.S., Song, H.H., Hong, J.M., Kwon, O.K., Jeon, C.M., Kim, J.H., Lee, S.W., Lee, J.K., Jin, H., Li, W.Y., Oh, S.R., Hahn, K.W., and Ahn, K.S. (2015). Callicarpa japonica Thunb. reduces inflammatory responses: a mouse model of lipopolysaccharide-induced acute lung injury. Int. Immunopharmacol 26(1): 174-180.

Spencer, J.P., and Crozier, A. (2012). Flavonoids and Related Compounds: Bioavailability and Function. CRC Press, Boca Raton, FL, USA

Tewtrakul, S., and Subhadhirasakul, S. (2008). Effects of compounds from Kaempferia parviflora on nitric oxide, prostaglandin E2 and tumor necrosis factor- $\alpha$ productions in RAW264.7 macrophage cells. J. Ethnopharmacol. 120(1): 81-84

Tewtrakul, S., Subhadhirasakul, S., Karalai, C., Ponglimanont, C., and Cheenpracha, S. (2009). Anti-inflammatory effects of compounds from Kaempferia parviflora and Boesenbergia pandurata. Food Chem. 115(2): 534-538.

Uckoo, R.M., Jayaprakasha, G.K., Vikram, A., and Patil, B.S. (2015). Polymethoxyflavones isolated from the peel of Miaray Mandarin (Citrus miaray) have biofilm inhibitory activity in Vibrio harveyi. J. Agric. Food Chem. 63(32): 7180-7189.

Wang, R., Li, S., and Ho, C-T. (2018). Absorption of polymethoxyflavones and their derivatives. J. Food Bioact. 2: 82-90.

Wang, Y., Xin, X., Jin, Z., Hu, Y., Li, X., Wu, J., and Jin, M. (2011). Anti-diabetic effects of pentamethylquercetin in neonatally streptozotocininduced diabetic rats. Eur. J. Pharmacol. 668(1-2): 347-353.

Wu, X., Song, M., Qiu, P., Rakariyatham, K., Li, F., Gao, Z., Cai, X., Wang, M. Xu, F., Zheng, J., and Xiao, H. (2017). Synergistic chemopreventive effects of nobiletin and atorvastatin on colon carcinogenesis. Carcinogenesis. 38(4): 455-464.

Wu, J., Zhao, Y.M., and Deng, Z.K. (2018). Tangeretin ameliorates renal failure via regulating oxidative stress, NF-KB-TNF- $\alpha$ /iNOS signalling and improves memory and cognitive deficits in 5/6 nephrectomized rats. Inflammopharmacology 26(1): 119-132.

Xiong, Y., Chen, D., Yu, C., Lv, B., Peng, J., Wang, J., and Lin, Y. (2015). Citrus nobiletin ameliorates experimental colitis by reducing inflammation and restoring impaired intestinal barrier function. Mol. Nutr. Food Res. 59(5): 829-842.

Xu, J.J., Liu, Z., Tang, W., Wang, G.C., Chung, H.Y., Liu, Q.Y., Zhuang, L., Li, M.M., and Li, Y.L. (2015). Tangeretin from Citrus reticulate inhibits respiratory syncytial virus replication and associated inflammation in vivo. J. Agric. Food Chem. 63(43): 9520-9527.

Yasunaga, S., Domen, M., Nishi, K., Kadota, A., and Sugahara, T. (2016). Nobiletin suppresses monocyte chemoattractant protein-1 (MCP1) expression by regulating MAPK signaling in 3T3-L1 cells. J. Funct. Foods. 27: 406-415.

Yuasa, K., Tada, K., Harita, G., Fujimoto, T., Tsukayama, M., and Tsuji, A. (2012). Sudachitin, a polymethoxyflavone from Citrus sudachi, suppresses lipopolysaccharide-induced inflammatory responses in mouse macrophage-like RAW264 cells. Biosci. Biotechnol. Biochem. 76(3): 598-600.

Zhang, G., and Ghosh, S. (2000). Molecular mechanisms of NF-kB activation induced by bacterial lipopolysaccharide through Toll-like receptors. J. Endotoxin Res. 6(6): 453-457.

Zhang, N., Yang, Z., Xiang, S.Z., Jin, Y.G., Wei, W.Y., Bian, Z.Y., Deng, W., and Tang, Q.Z. (2016a). Nobiletin attenuates cardiac dysfunction, oxidative stress, and inflammatory in streptozotocin: induced diabetic cardiomyopathy. Mol. Cell Biochem. 417(1-2): 87-96.

Zhang, L., Zhang, X., Zhang, C., Bai, X., Zhang, J., Zhao, X., Chen, L., Wang, L., Zhu, C., Cui, L., Chen, R., Zhao, T., and Zhao, Y. (2016b). Nobiletin promotes antioxidant and anti-inflammatory responses and elicits protection against ischemic stroke in vivo. Brain Res. 1636: 130-141. 